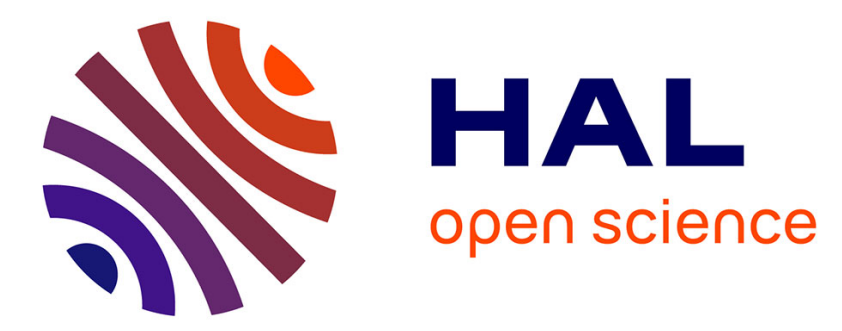

\title{
Full-Wave Indoor Measurements' Cross-Validation With the Model Demos for Foliage Penetrating Applications
}

\author{
Lydia Hettak, Hassan Saleh, Cyril Dahon, Massimiliano Casaletti, Olivier
}

Meyer, Jean-Michel Geffrin, Hélène Roussel

\section{- To cite this version:}

Lydia Hettak, Hassan Saleh, Cyril Dahon, Massimiliano Casaletti, Olivier Meyer, et al.. Full-Wave Indoor Measurements' Cross-Validation With the Model Demos for Foliage Penetrating Applications. IEEE Geoscience and Remote Sensing Letters, 2020, 17 (6), pp.933. 10.1109/LGRS.2019.2940141 . hal-02474268

\section{HAL Id: hal-02474268 \\ https: / hal.sorbonne-universite.fr/hal-02474268}

Submitted on 13 Feb 2020

HAL is a multi-disciplinary open access archive for the deposit and dissemination of scientific research documents, whether they are published or not. The documents may come from teaching and research institutions in France or abroad, or from public or private research centers.
L'archive ouverte pluridisciplinaire HAL, est destinée au dépôt et à la diffusion de documents scientifiques de niveau recherche, publiés ou non, émanant des établissements d'enseignement et de recherche français ou étrangers, des laboratoires publics ou privés. 


\title{
Full wave indoor measurements cross-validation with the model DEMOS for Foliage Penetrating applications
}

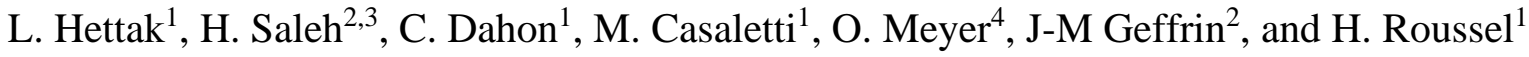

\begin{abstract}
For Foliage Penetrating (FoPen) radar development we developed previously a hybrid volume/surface model, named DEMOS, to evaluate the electromagnetic scattering from large scenes composed by targets (metallic objects) placed in a natural environment (dielectric object). In this paper, we compare the scattered field obtained by DEMOS with quasi-monostatic measurements done in an anechoic chamber on scaled models composed of dielectric and metallic structures. For all measurements, we consider both polarizations, $\mathrm{HH}$ and VV. Our final objective is to determine the optimal configurations for the detection of a target placed in a forest environment in the VHF-UHF frequency bands.
\end{abstract}

Index Terms-Monostatic measurements, radar detection, Foliage Penetrating.

\section{INTRODUCTION}

FoPen (Foliage Penetrating) radar development is very expensive because it requires several measurement campaigns to estimate the best radar configuration for target detection (incident angle, polarization, etc...). This justifies the interest in "full wave" electromagnetic models dedicated to the modeling of scattering of large forest areas in the presence of metallic targets or a human [1]-[2]. Such models could replace in the near future the first step of radar design thus limiting the number of measurement campaigns. Nevertheless, the implementation of such an electromagnetic model presents two main challenges. First, this requires taking into account a scene composed of highly heterogeneous media (lossy dielectric materials for the trees and metals for the target). Second, a large-sized scene (the focal spot of the UHF radar antenna could be up to $50 \times 50 \mathrm{~m}^{2}$ ). Several full-wave techniques can be used to evaluate the field scattered by a mixed environment (dielectric and metallic media). In our case, we take advantage of an integral formulation that combine the volume integral equation (VIE) for the trees [3] with the surface ones (SIE) for the targets [4] and use the same Dyadic Green's function for both formulations to incorporate the interface air/ground [5]. We named our model DEMOS for Domain dEcomposition MOdel for the analysis of target Scattering in forested areas. This model has been numerically validated in the first step for a simple configuration with the commercial software FEKO [5].

Here we compare in amplitude and phase our model DEMOS with quasi-monostatic measurements performed in an anechoic chamber on scaled models combining metallic and dielectric elements. In order to be able to consider realistic scenes, a scale

This work was supported by the French Agence Nationale de la Recherche (A.N.R.) and the French Direction Générale de l'Armement (D.G.A.) under the project MOBILE.

${ }^{1}$ L. Hettak, C. Dahon, M. Casaletti and H. Roussel are with Sorbonne Université, L2E, F-75005 Paris, France. helene.roussel@ sorbonne-universite.fr

${ }^{2}$ J-M Geffrin and H. Saleh are with Aix Marseille Univ, CNRS, Centrale Marseille, Institut Fresnel, Marseille, France. factor of a twenty-fifth has been used on both geometry and frequency band of interest. This choice transforms the FoPen frequency band $(320-480 \mathrm{MHz})$ into $(8-12 \mathrm{GHz})$ fitting the operative bandwidth of our measurement platform, and allowing the measurement of scenes containing an electrically large target and trees.

This approach, already validated for forested areas [6]-[7] is now extended to the case of targets placed in a forest environment. The aim of this paper is to present crossvalidations of our model DEMOS with measurements in an anechoic chamber.

\section{FORMULATION}

In order to consider realistic forest scenes and to overcome the limitations due to the huge number of unknowns, an ExtendedCBFM (CBFM-E) compression scheme has been implemented in our model (DEMOS) [5]. The algorithm begins with the decomposition of the computational domain associated with the forest (trunks and branches) into blocks and proceeds to generate the characteristic basis functions (CBFs) for these blocks. In a second step, the number of CBFs retained for each block is much lower than the original number of low-level basis functions for the same block to obtain a significant compression ratio (ratio between the number of the original basis functions and the number of post-CBFM unknowns). This turns out in a significant reduction in terms of computational time and memory consumption compared to a conventional MoM (Method of Moments) approach [8].

\section{MEASUREMENTS ON SCALED MODELS}

Since environmental conditions and experimental uncertainties may dramatically influence the scattering, as well as the forest electromagnetic properties change during the year, the underlying idea is to carry out scaled-model measurements in an anechoic chamber. The measurements are made using the quasi-monostatic configuration in the anechoic chamber of the CCRM [9, 10]. Radar scattering models for a metal target existing in a forest environment are usually designed to operate in the VHF band between 320 and $480 \mathrm{MHz}$. The microwave analog situation is reduced with a scaling factor of $1 / 25$ and the experiments are made in the frequency band from 8 to $12 \mathrm{GHz}$. The ground is represented by a flat metal plate with $1 \mathrm{~m}$ diameter, that is considered to be infinite in the model. The

\footnotetext{
${ }^{3} \mathrm{H}$. Saleh was also with Centre Commun de Ressources en Microondes (CCRM), 5 Rue Enrico FERMI, Technopôle de château Gombert, 13012, Marseille, France

${ }^{4}$ O. Meyer is with GeePs, UMR CNRS 8507, Centrale Supélec, Univ. ParisSud, Université Paris-Saclay,Sorbonne Universités, UPMC Univ Paris 6, Gifsur-Yvette, France
} 
metal plate is glued on expanded polystyrene support, which is placed on the top of the central mast. The metal plate is present during the measurement of the total and incident fields. The targets to be measured are therefore aligned at the center of the metal plate. The measurements are made using X-band horn antennas and the residual bistatisme angle between them is $12^{\circ}$. The distance between the antennas and the origin $\mathrm{O}$ at the center of the metal plate is $1.648 \mathrm{~m}$, which is around $41 \mathrm{~m}$ at the initial scale. The displacement of the wagon carrying the antennas on the vertical arch is denoted by the angle $\varphi_{s}$. The measurements are made in the two co-polarization cases $\theta \theta(V V)$ and $\varphi \varphi(H H)$ defined in Figure 1 and Figure 2. A metal squat cylinder was chosen as a reference target to calibrate the measured scattered field. The cylinder has a diameter of $95 \mathrm{~mm}$ and a height of $44.45 \mathrm{~mm}$. During the measurement, the center of the cylinder is aligned at the center of the metal plate on one of its two parallel faces. It was chosen for two reasons: first, it has a relatively high electromagnetic signature which can overcome the experimental noise. Second, it is asymmetric in the measurement configuration so that its scattered field is variable with respect to the monostatic angle $\varphi_{s}$.
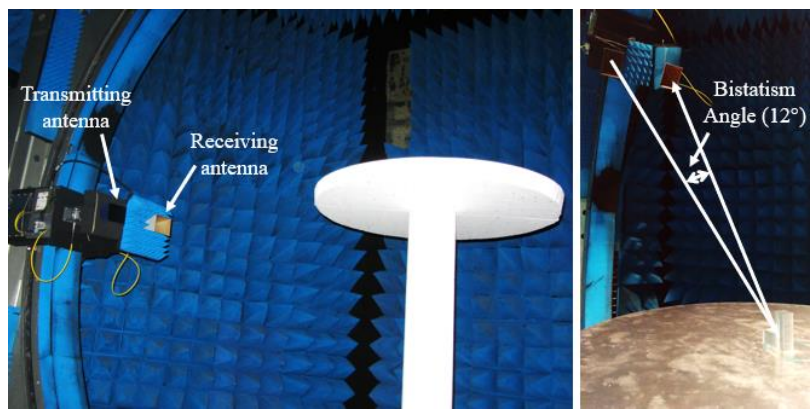

Figure 1: In the case of this picture the emitter and the receiver are moved on a semi-vertical arch during the scattering measurement in a quasi-monostatic configuration. The targets are placed on the metal disk supported by a polystyrene mast.

Our first comparison concerns a simple configuration composed of an L-shaped target placed on a metal plate (see Figure 2). In figure 3, we compare for the $\mathrm{VV}$ and $\mathrm{HH}$ polarizations the variations of the measured and computed fields with respect to the incident angle $\theta$ at $8 \mathrm{GHz}$ and $11 \mathrm{GHz}$. We obtain a good agreement between the two results both in amplitude and phase.
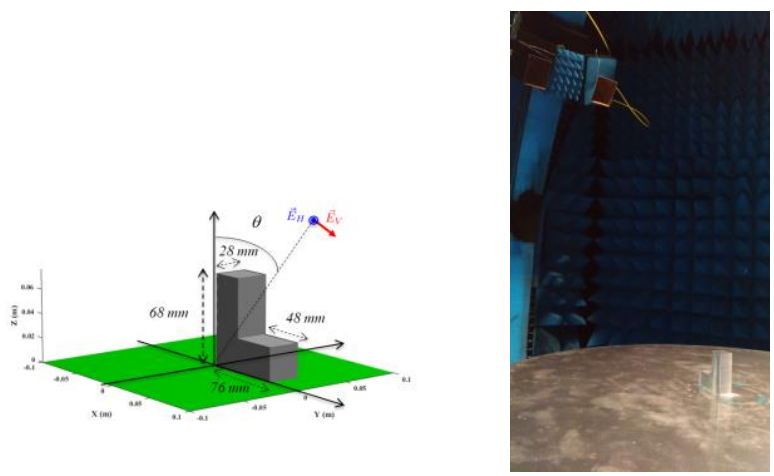

Figure 2: Geometry of the L-shaped target and image of the measurement
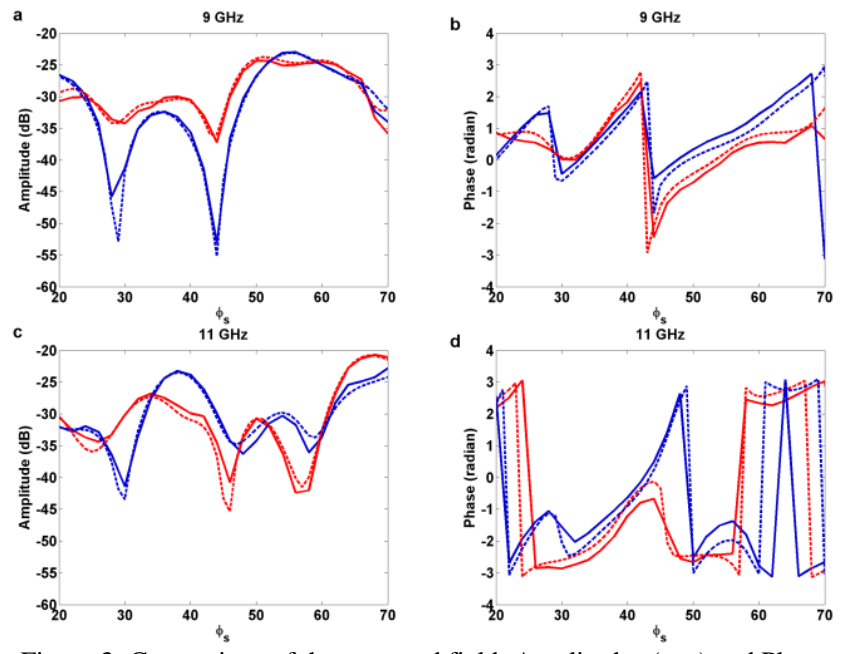

Figure 3: Comparison of the scattered field: Amplitudes (a, c) and Phases (b, d) of the measured (dashed) and calculated (solid) scattered fields by the L-shaped target placed on a metal plate for VV (blue) polarization and $\mathrm{HH}$ (red) polarization at $9(\mathrm{a}, \mathrm{b})$ and $11 \mathrm{GHz}(\mathrm{c}, \mathrm{d})$.

If we look at the holographic view of the field in figure 4, we observe a globally good agreement between measurements and simulations for both polarizations.

a)

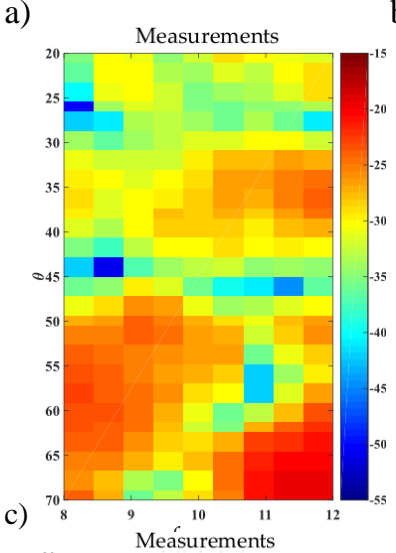

b)
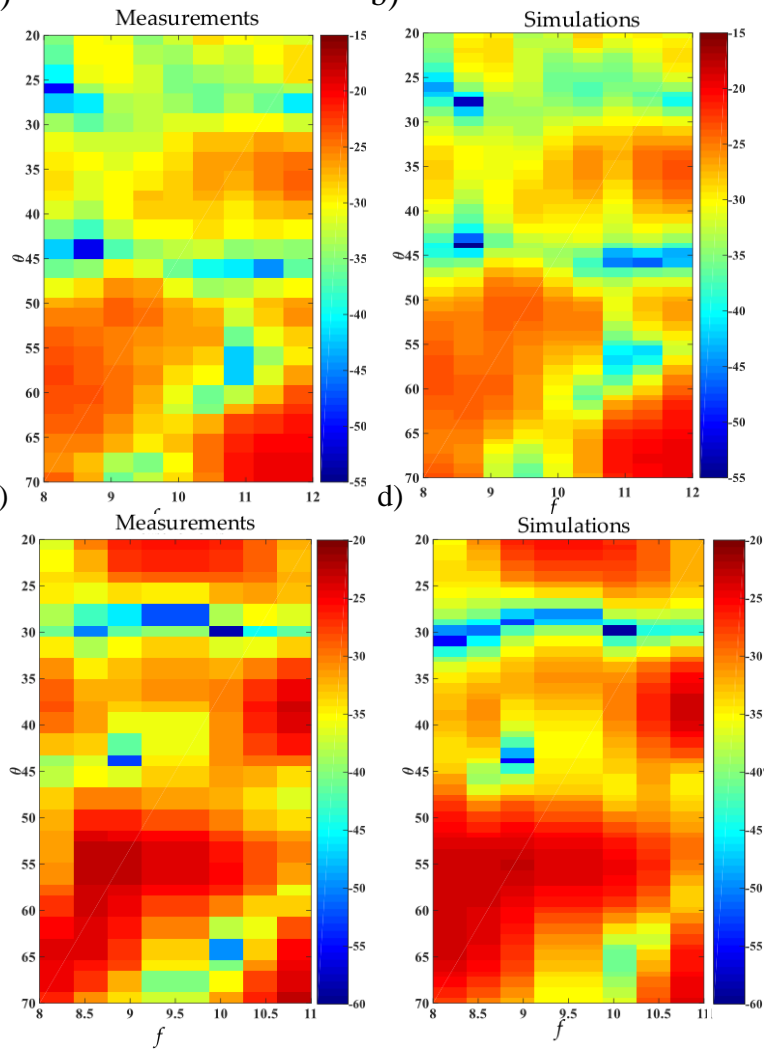

Figure 4: Holographic views for the frequency band 8-11 GHz and $\theta$ varying from 20 to $70^{\circ}$ (measurements left and simulations right) $\mathrm{VV}$ polarization (a, b) and $\mathrm{HH}$ polarization $(\mathrm{c}, \mathrm{d})$

For the second case, we consider the same metallic target associated with a high permittivity dielectric cylinder. Given that it is not simple to obtain a cylinder of high permittivity corresponding to wood permittivity [11], we have chosen to make the dielectric cylinders ourselves, first we printed with a 
3D printer a hollow cylinder in ABS and in a second phase we filled it with a liquid composed of a $76 \%$ glycerol part and of $24 \%$ water. The variation of the permittivity of this liquid has been measured at $25^{\circ} \mathrm{C}$ in the frequency band $8-12 \mathrm{GHz}$. The dielectric characterization system consists of a junction between a coaxial waveguided connected to an Agilent E8364C vector network analyzer on a side and a cylindrical cell at the other side. This cell is made up of a circular $7 \mathrm{~mm}$ diameter waveguide filled up by a liquid under test, terminated by a short circuit [12]. A dielectric coaxial tight window is inserted between the waveguide and the cell. The device is connected to the vector network analyzer [13-14]. The real part of the relative permittivity varies from eight to ten and the imaginary part from six to nine. The height of the cylinder is $16 \mathrm{~cm}$ which corresponds to $4 \mathrm{~m}$ for the transposed size. This height is so comparable to that of a tree of medium height. Since we want to study the masking effect of the target by the trees, we measured the scattered field for three positions of the cylinder: ahead, beside and behind the target (Figure 5).
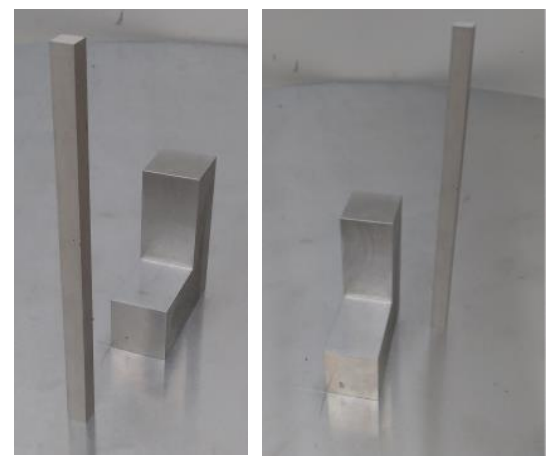

Figure 5: View of two measured configurations (ahead and beside)

In figure 6 we compare for the $\mathrm{HH}$ and $\mathrm{VV}$ polarizations the variations of the measured and scattered fields with respect to the incident angle $\theta$ at $8 \mathrm{GHz}$ and $11 \mathrm{GHz}$ when the cylinder is beside and behind the target respectively. We notice a good agreement between measurements and simulations since the permittivity of the dielectric bar is not perfectly mastered.

a)
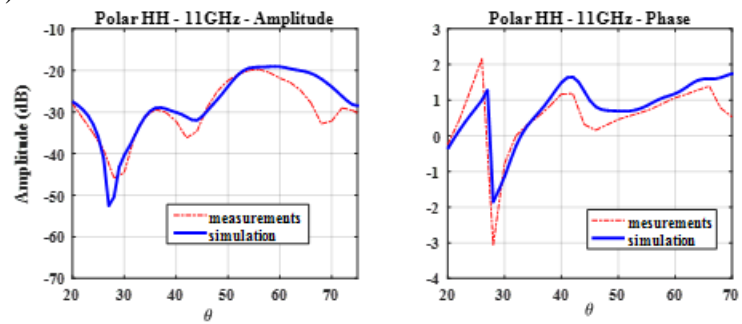

b)
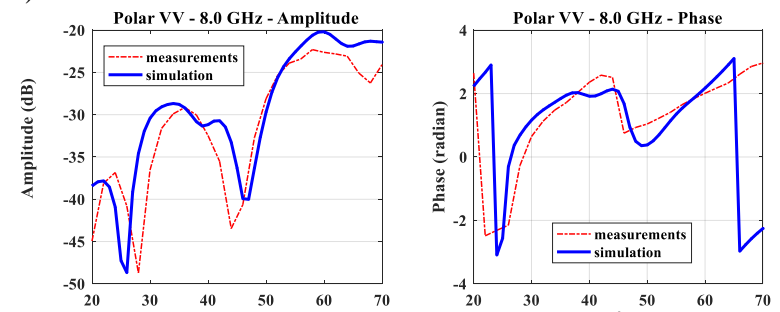

Figure 6: Comparison of the scattered field: Amplitudes and Phases measured (red) and calculated (blue) scattered fields by the L-shaped target and a dielectric cylinder placed on a metal plate for $\mathrm{VV}$ polarization at $8 \mathrm{GHz}$ (b) and $\mathrm{HH}$ polarization at $11 \mathrm{GHz}$ (a) when the cylinder is beside (a) or behind (b) the target.
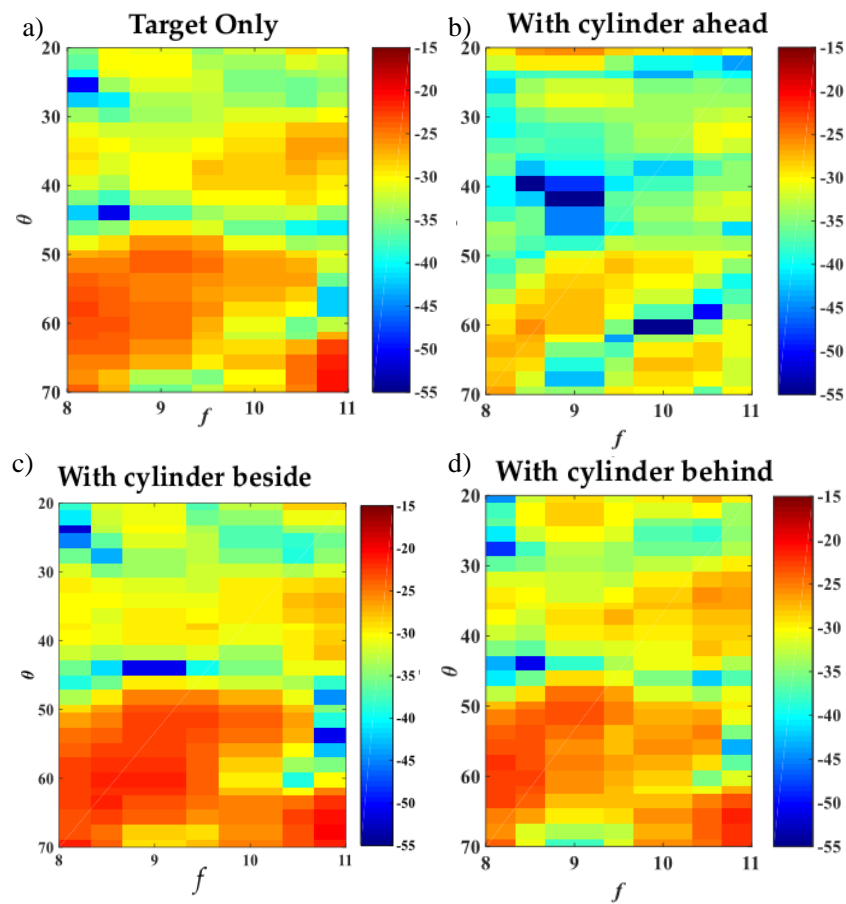

Figure 7: Holographic views of measurements in VV polarization for the frequency band $8-11 \mathrm{GHz}$ and $\theta$ varying from $20^{\circ}$ to $70^{\circ}$ for the 4 configurations: a) Target only, b) Position 1 (target with cylinder ahead), c) Position 2 (target with cylinder beside) and d) Position 3 (target with cylinder behind)

In figure 7 and 8 we give the variations of the measured scattered field for both co-polarization $\mathrm{VV}$ and $\mathrm{HH}$, with respect to the incident angle $\theta$ and frequency when we consider four different configurations: the target only, the target with the cylinder ahead, beside and behind. We compare only measurements in order to evaluate the masking effect of the cylinder. We observe that when the cylinder is ahead of the target, the level of the field diffracted by the target is strongly attenuated for the VV polarization. On the other hand, for the $\mathrm{HH}$ polarization, this masking effect is less pronounced. Furthermore, the level of the diffracted field by the target is stronger for angles beyond $50^{\circ}$. This observation was reproduced on a measurement made on a much more complex configuration composed of a model of tank placed in the middle of a set of five trees with branches (see figure 9). These measurements (figure 10) suggest that the $\mathrm{HH}$ polarization will facilitate the detection of hidden targets undercover and that the observation angles above $50^{\circ}$ should be preferred. 


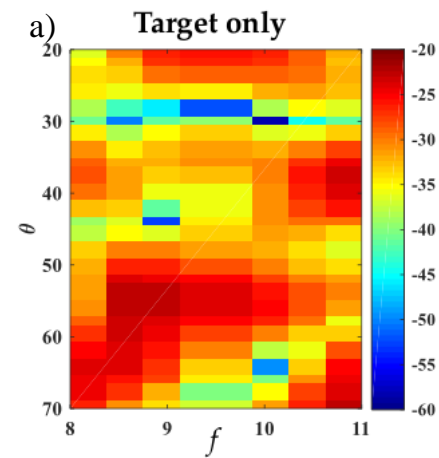

c) With cylinder beside

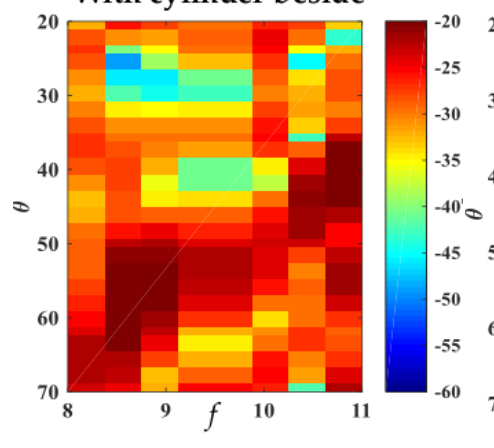

d) With cylinder behind

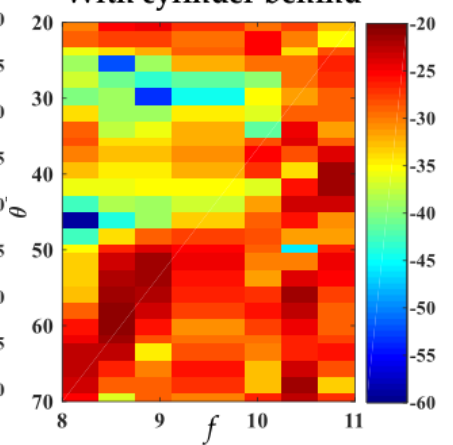

Figure 8: Holographic views of measurements in $\mathrm{HH}$ polarization for the frequency band $8-11 \mathrm{GHz}$ and $\theta$ varying from $20^{\circ}$ to $70^{\circ}$ for the 4 configurations: a) Target only, b) Position 1 (target with cylinder ahead), c) Position 2 (target with cylinder beside) and d) Position 3 (target with cylinder behind)

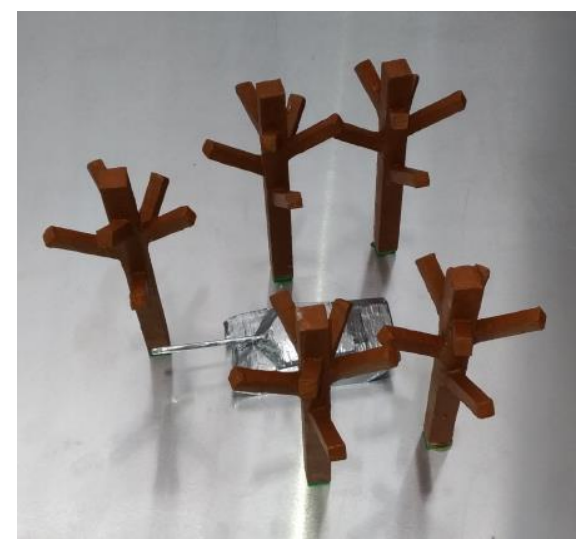

Figure 9: View of scaled tank placed in the middle of the five scaled trees a)

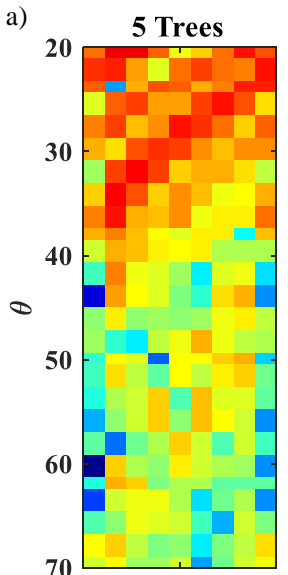

b) Tank

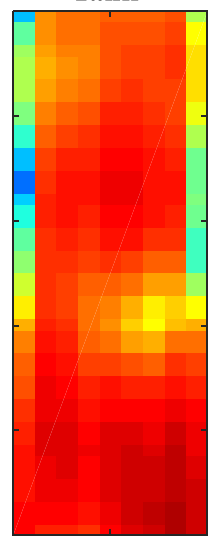

d)

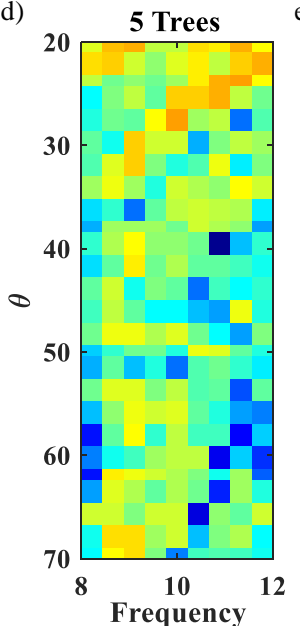

e)

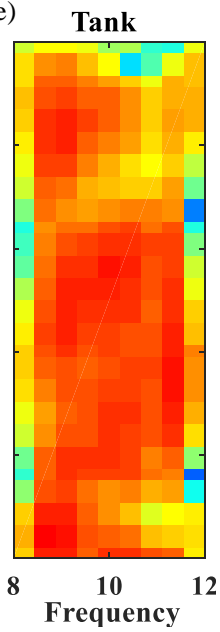

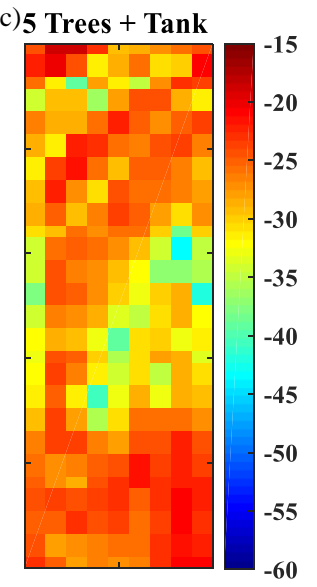

f) 5 Trees + Tank

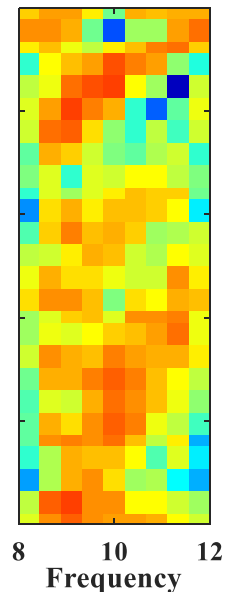

Figure 10: Holographic views of measurements for the frequency band 8-12 $\mathrm{GHz}$ and $\theta$ varying from $20^{\circ}$ to $70^{\circ}$ for the tank placed in the middle of 5 trees Legend : (a, d) Only the 5 trees (b, e) only the tank, (c, d) the tank and the 5 trees $(\mathrm{a}, \mathrm{b}, \mathrm{c}) \mathrm{HH}$ polarization $(\mathrm{d}, \mathrm{e}, \mathrm{f})$ and $\mathrm{VV}$ polarization.

\section{CONCLUSION}

We propose in this paper to compare a hybrid MoM formulation that considers metallic targets inside a forest environment with measurements in an anechoic chamber. In a first step, we give validation of our model with measurements and in a second step an interpretation on measurements on scaled models. In the future, we will consider bi-static configurations and cross polarisations for measurements.

\section{ACKNOWLEDGMENT}

This work is supported by the French Agence Nationale de la Recherche (ANR) and the French Direction Générale de l'Armement (DGA) under the Project MOBILE.

\section{REFERENCES}

[1] M. Dehmollaian and K. Sarabandi, "Electromagnetic Scattering From Foliage Camouflaged Complex Targets," in IEEE Transactions on Geoscience and Remote Sensing, vol. 44, no. 10, pp. 2698-2709, Oct. 2006.

[2] D. Liao, "Application of Discrete Scatterer Technique for Scene Response Estimation in FOPEN Radar Simulations," in IEEE 
Geoscience and Remote Sensing Letters, vol. 14, no. 8, pp. 13431347, Aug. 2017.

[3] I. Fenni, H. Roussel, M. Darces and R. Mittra, "Fast Analysis of Large 3-D Dielectric Scattering Problems Arising in Remote Sensing of Forest Areas Using the CBFM," in IEEE Transactions on Antennas and Propagation, vol. 62, no. 8, pp. 4282-4291, Aug. 2014.

[4] S. Rao, D. Wilton and A. Glisson, "Electromagnetic scattering by surfaces of arbitrary shape," in IEEE Transactions on Antennas and Propagation, vol. 30, no. 3, pp. 409-418, May 1982.

[5] L. Hettak, H. Roussel, M. Casaletti and R. Mittra,"A Numerically Efficient Method for Predicting the Scattering Characteristics of a Complex Metallic Target Located Inside a Large Forested Area," in IEEE Transactions on Geoscience and Remote Sensing, vol. 56, no. 2, pp. 1177-1185, Feb. 2018.

[6] S. Bellez,, C. Dahon and H. Roussel, Juan Carlos Castelli, Anil Cheraly. "Full polarimetric bistatic radar imaging experiments on sets of dielectric cylinders above a conductive circular plate." In IEEE Transactions on Geoscience and Remote Sensing, 2013, 51 (7), pp.4164-4176.

[7] S. Bellez, H. Roussel, C. Dahon and J-M. Geffrin,"A rigorous forest scattering model validation through comparison with indoor bistatic scattering measurements." In Progress In Electromagnetics Research B, 2011, 33, pp. 1-19.

[8] I. Fenni, H. Roussel, M. Darces and R. Mittra, "Efficiency Enhancement of the Characteristic Basis Function Method for Modeling Forest Scattering Using the Adaptive Cross Approximation Algorithm." In IEEE Antennas and Propagation Society Newsletter, 2016, 64 (10), pp.4539 - 4544.

[9] H. Saleh, "Application of the microwave analogy to study the scattering properties of trees, atmospheric particles and microorganism.” PhD thesis, Aix-Marseille University, 2017.

[10] J.M. Geffrin, P. Sabouroux, P. and C. Eyraud, "Free-space experimental scattering database continuation: experimental set-up and measurement precision. ," in Inverse Problem, 21(6), 2015.

[11] D. Liao and T. Dogaru, "Large-scale, full-wave scattering phenomenology characterization of realistic trees," 2015 IEEE International Symposium on Antennas and Propagation \& USNC/URSI National Radio Science.

[12] N.-E. Belhadj-Tahar, A. Fourrier-Lamer, "Broad-Band Analysis of a Coaxial Discontinuity Used for Dielectric Measurements," in IEEE Trans. Microwave Theor. Tech. vol. 34, issue 3, pp 346-350, 1986.

[13] H. Acikgoz, Y. Le Bihan, O. Meyer and L. Pichon, "Neural networks for broad-band evaluation of complex permittivity using a coaxial discontinuity," in Eur. Phys. J. Appl. Phys. 39, 197-2007.

[14] O. Meyer, C. Gilbert, A. Fourrier-Lamer, H. Cachet, "In-Vitro Broad Band Impedance Study of a Biochemical Reaction under Nanopulses: Electrode Impedance as a Reaction Sensor," in Journal of The Electrochemical Society, vol. 161, no. 4, pp. B62-B69, 2014. 